\title{
DESIGN AND SIMULATION OF CROSS-BLOCK STRUCTURED RADAR ABSORBING METAMATERIAL BASED ON CARBONYL IRON POWDER COMPOSITE
}

\author{
M. B. Abdullahi' ${ }^{1}{ }^{*}$ and M. H. Ali ${ }^{2}$ \\ 1, DePARTMENT OF PhySiCS, USMANU DANFOdIYO UniVERSITY SOKOTO, SOKOTO STATE, NIGERIA. \\ 2, DEPARTMENT OF PHYSICS, BAYERO UNIVERSITY KANO, KANO STATE, NIGERIA. \\ E-mail addresses: ${ }^{1}$ abdullahi.bello@udusok.edu.ng, ${ }^{2}$ mutariali@gmail.com
}

\begin{abstract}
This paper reports the design and simulation of a three layered cross-block structured radar absorbing metamaterial (RAMM). The effective electromagnetic parameters of the designed structure are highly dependent on its geometric dimensions, subsequent/y behaving as a metamaterial. COMSOL Multiphysics simulation software was used to analyze the frequency dependent absorption response of the designed RAMM. The input impedance of multilayered absorber and strong fluctuation theory equations are used to theoretically verify the absorption response of the RAMM. The simulated result showed that the reflectivity of the RAMM is below $10 \mathrm{~dB}$ from $4.2-18.0 \mathrm{GHz}$ frequency band with absorber thickness of $4.2 \mathrm{~mm}$. The calculated reflectivity result is in close agreement with the simulated result, thus confirming the validity of the design. The operational bandwidth to thickness ratio of this RAMM was found to be 13.029 making it better than the recently reported one with a value of 9.745 and thus contributing significantly in overcoming the contradicting demand of broadband and thin thickness.
\end{abstract}

Keywords: Bandwidth, COMSOL Multiphysics, Metamaterial, Radar Absorbing Metamaterial, Reflectivity.

\section{INTRODUCTION}

Validation of three decades old theoretical prediction of negative index of refraction [1] through its experimental demonstration, initiated a new area of research known as metamaterials. Metamaterials are artificially designed structures capable of demonstrating fascinating and unique properties that are not found in naturally occurring materials such as perfect lensing [2-3], invisibility cloaking [4], negative index of refraction [5-8] and perfect absorption [9]. Interests in metamaterials originate from their available use in virtually any band of the electromagnetic spectrum and their ability to achieve almost any desired response [10]. Since the inspiration by Landy et al [11] in the last decade, metamaterial absorbers research witnessed rapid transitional development from the single band, fixed polarization and normal incidence to multiband, broadband, polarization insensitive and wide angle of incidence nowadays [12].

\footnotetext{
* Corresponding author, tel: +234 - 706-817 - 9649
}

Metamaterial absorbers (MMA) operate in a specific narrow frequency band which limits their application in broadband requirement [13]. MMA that include magnetic microwave absorbing materials (MMWAMs) as substrates, obviously improved the narrow band characteristic of the MMAs [14-15] primarily due to its large value of permeability [16]. MMWAMS demonstrates light weight and thin thickness in the frequency regime of $2-18 \mathrm{GHz}$ [17-20], the most exploited band in radio frequency due to its wide application in radar and other vital communication devices [21]. As thin as $1 \mathrm{~mm}$ thickness, they can absorb more than $85 \%$ of the incident electromagnetic wave over the $8-18 \mathrm{GHz}$ range [2223]. However, the bandwidth for $90 \%$ absorption is limited to a few $\mathrm{GHz}$ as the absorption in $2-8 \mathrm{GHz}$ is very weak, hence obtaining a wide bandwidth response without compromising thickness is a great challenge [24-26].

Previously, inclusion of magnetic substrates in the design of MMA has been widely adopted. A composite 
X-band radar absorbing structure of $2.93 \mathrm{~mm}$ thickness that absorbed more than $90 \%$ of incident wave was proposed [27]. A new type of thin and broadband three layers structure metamaterial absorbers [28] that achieved in excess of $12 \mathrm{GHz}$ absorption bandwidth with reflectivity below $-10 \mathrm{~dB}$ in the $2-18 \mathrm{GHz}$ frequency range at a thickness of $1.8 \mathrm{~mm}$ was reported. Likewise, a three-layered metamaterial absorber [29] intended for broadband radar absorption was fabricated by means of selective laser sintering 3D printing technology and optimized results indicated reflectivity below $-10 \mathrm{~dB}$ in the frequency range from $8 \mathrm{GHz}$ to $18 \mathrm{GHz}$ at $2.89 \mathrm{~mm}$ thickness. A composite absorbent consisting of carbonyl iron powder and nylon [30]was used to modeled a metamaterial absorber of $4.7 \mathrm{~mm}$ optimized thickness which results in a reflection loss below $-10 \mathrm{~dB}$ from 4 to $18 \mathrm{GHz}$ range.

Although the MMA with magnetic inclusion exhibits broadband absorption characteristics [29-30], it is still required to improve broadband absorption at decreased thickness. In this work, a flaked carbonyl iron powder magnetic microwave absorbing material was structured and its geometric dimensions optimized. This fine tunes its absorption properties hence giving it metamaterial concept and characteristics leading to a competitive operational bandwidth to thickness ratio value.

\section{THEORY}

Generally, the reflectivity of an absorber R, backed by perfect electrical conductor (PEC) ground plate can be written as [30]

$$
R=20 \log \left|\frac{Z_{\text {in }}^{0}-1}{Z_{\text {in }}^{0}+1}\right|
$$

where $R$ is reflectance and $Z_{i n}{ }^{0}$ is surface layer input impedance.

The governing equation of a multilayered absorber based on transmission line method which is required to evaluate the reflectivity of the model absorber is presented as [30]

$$
Z_{\text {in }} i=Z_{i} \frac{Z_{\text {in }}(i+1)+Z_{i} \tanh \gamma_{i} d_{i}}{Z_{i}+Z_{\text {in }}(i+1) \tanh \gamma_{i} d_{i}}
$$

where $Z_{i n} i=$ Input impedance, $d_{i}=$ Layer thickness, $Z_{i}=$ Characteristic impedance, $\gamma_{i}=$ Propagation constant, $i=$ layer number and $Z_{\text {in }} 4=0 . Z_{i}=\sqrt{\frac{\mu_{r, i}}{\varepsilon_{r, i}}}$ and $\gamma_{i}=j 2 \pi f \frac{\sqrt{\varepsilon_{r, i} \mu_{r, i}}}{c}$.

Effective electromagnetic parameters of structured layers can be obtained using equation (3), which is based on strong fluctuation theory of honeycombstructured surfaces [31].

$$
\begin{aligned}
\varepsilon_{e f f}=\frac{1}{2}[(1-2 g) & \left(1-\varepsilon_{r}\right) \\
+ & \left.\sqrt{(1-2 g)^{2}\left(1-\varepsilon_{r}\right)^{2}+4 \varepsilon_{r}}\right]
\end{aligned}
$$

Here, $\varepsilon_{e f f}$ is the effective permittivity, $g$ is the volume ratio of the solid part in a layer, and $\varepsilon_{r}$ is the permittivity of the solid. Effective permeability $\mu_{e f f}$ can be obtained by replacing $\varepsilon_{r}$ with $\mu_{r}$ in equation 3 . The operational bandwidth to thickness ratio that was used to describe the performance of the absorber is given by [32]

$$
\frac{\text { Bandwith }}{\text { Thicknes }}=\frac{\lambda_{\text {fmin }}-\lambda_{\text {fmax }}}{h}
$$

where $\lambda_{\text {fmin }}$ and $\lambda_{\text {fmax }}$ are the wavelengths at $f_{\min }$ and $f_{\max }$ respectively and $h$ is the thickness of the absorber.

\section{DESIGN AND SIMULATION}

The three dimensional optimized geometric structure of a single unit cell of the proposed radar absorbing metamaterial (RAMM) is shown in Figure 1. It consists of three layers of flaked carbonyl iron powder materials with a copper plate ground plane. The surface layer is cross shaped, the middle layer is block shape structured and the bottom layer is considered as a conventional single slab absorber. The thickness of the bottom copper metallic plate $(0.036 \mathrm{~mm})$ is designed to be greater than the skin depth of the copper under the studied frequency intervals (2-18 $\mathrm{GHz}$ ), so that it does not transmit any radiation. Finite element method (FEM) based on Comsol Multiphysics simulation package is used for numerical modeling of the proposed radar absorbing metamaterial.

The metamaterial absorber design in this work is for radar applications, therefore the frequency range of $2-18 \mathrm{GHz}$ is considered. The electromagnetic parameters of the flaked carbonyl iron powder as measured by [28] are imported into the simulation software to define the frequency dependent material properties using interpolation tool. The boundary conditions along $x$ - and $y$-axis are chosen to be perfect magnetic conductor (PMC) and perfect electric conductor (PEC) in order, where port is used to input plane polarized electromagnetic wave along z-axis. These boundary conditions as cited in [33] are used to mimic the infinite periodic structure that ensures symmetry along $x$ - and $y$-axis and the wave strikes perpendicularly onto the infinite radar absorbing metamaterial along z-axis. 


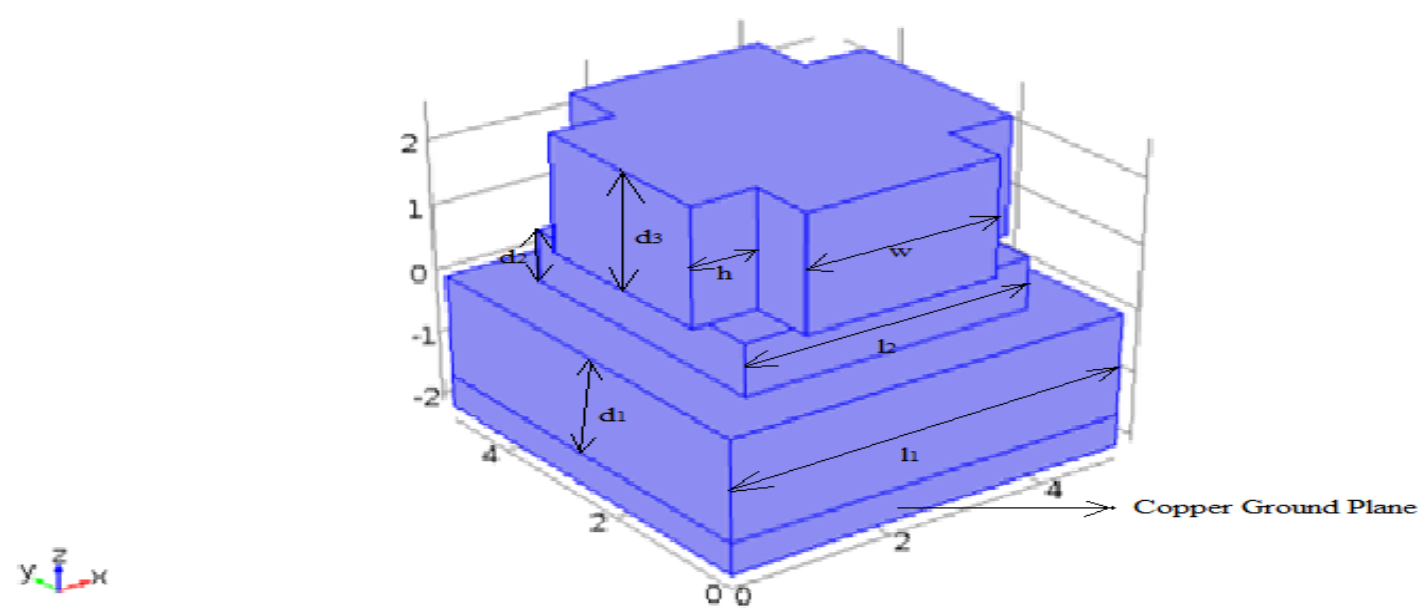

Figure 1: Designed Unit Cell Structure of the Proposed Radar Absorbing Metamaterial.

Geometric dimensions are: $l_{1}=5.0 \mathrm{~m}, l_{2}=3.6 \mathrm{~mm}, w=2.4 \mathrm{~mm}, h=0.8 \mathrm{~mm}, d_{1}=1.6 \mathrm{~mm}, d_{2}=0.8 \mathrm{~mm}, d_{3}=$ $1.8 \mathrm{~mm}$ and ground plane thickness $=0.036 \mathrm{~mm}$.

Physics controlled tetrahedral meshing is used in the simulation and impedance boundary condition (IBC) is selected to represent ground plane condition while the output of the simulation software is the scattering parameters (S-parameters).

In order to validate the COMSOL Multiphysics software used in this work, a metamaterial absorber which was numerically and experimentally realized by Zhou et al [29] using finite integration technique (FIT) based commercial program CST Microwave Studio, has been replicated on the COMSOL Multiphysics solver. The optimized unit cell geometric dimensions of the absorber as reported are $l_{1}=5.0 \mathrm{~m}, l_{2}=3.96 \mathrm{~mm}, l_{3}=$ $2.34 \mathrm{~mm}, d_{1}=0.84 \mathrm{~mm}, d_{2}=0.88 \mathrm{~mm}, d_{3}=1.17 \mathrm{~mm}$, ground plane thickness $=0.036 \mathrm{~mm}$. Their model

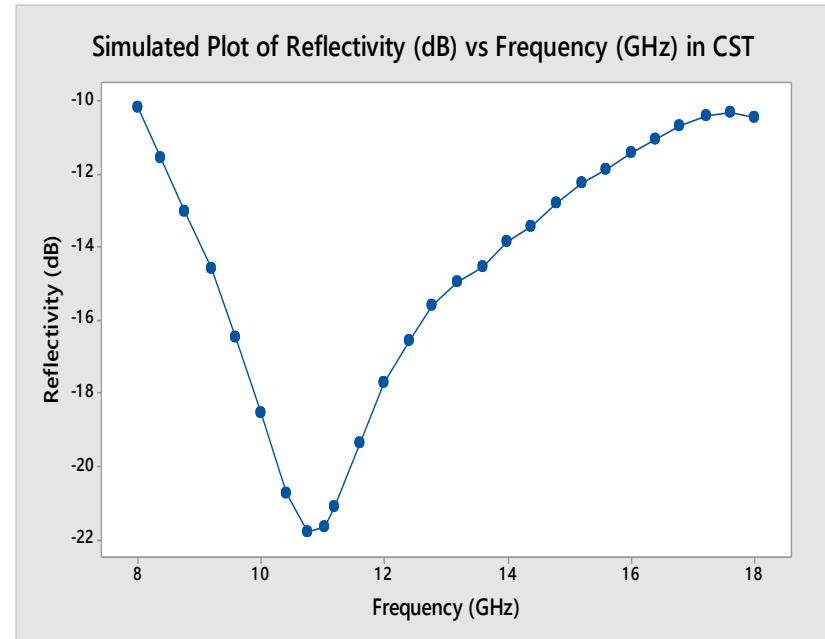

Figure 2: Simulation result using CST by Zhou, et. al [29] absorber demonstrates reflectivity below $-10 \mathrm{~dB}$ from $8-18 \mathrm{GHz}$ at $2.89 \mathrm{~mm}$.

The reflectivity of the absorber was theoretically evaluated using equations 2 and 3 . The calculated reflectivity is then compared with the simulated reflectivity in order to validate the performance of the designed RAMM.

\section{RESULTS AND DISCUSSIONS}

Simulation results of the radar absorbing metamaterial proposed by Zhou et al, is represented in Figure 2 . Figure 3 depicts the simulated result of the replicated metamaterial absorber reported by Zhou et al using COMSOL Multiphysics.

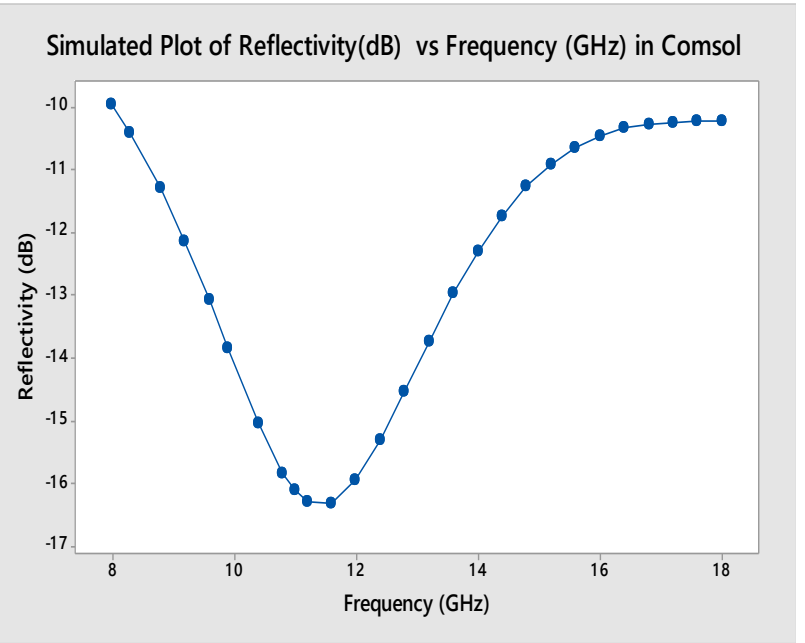

Figure 3: Simulation Results using COMSOL Multiphysics

Vol. 38, No. 4, October 2019 
The simulation results for both methods (FIT based solver by Zhou et a/ and FEM based solver used in this work) achieved matching results of below $-10 \mathrm{~dB}$ reflectivity throughout the $8-18 \mathrm{GHz}$ frequency range as could be seen from Figures 2 and 3, which is a confirmation of the precision and accuracy of COMSOL Multiphysics numerical solver used in the present work. The similarity of the reflectivity curves further confirms the accuracy of our simulation software despite slight deviations in the reflectivity peaks.

Parametric studies with respect to the dimensions of the structure are carried out to achieve optimal result for the model RAMM. The magnitude of the reflectivity as a function of the side lengths ( $w$ and $h$ ) of crossshaped layer and the layers thickness (parameter $d_{i}$ ) are separately presented in Figures 4 and 5 respectively. The parameters combination with optimum absorption characteristics are used in modeling the RAMM.

The reflectivity results from the simulations and theoretical calculations are shown in Figures 6 and 7. These results are compared in Figure 8. It is observed that, the simulated result of the designed RAMM has reflectivity below $-10 \mathrm{~dB}$ ranging between $4.2-18.0$ $\mathrm{GHz}$. This means the absorption of the incident radar wave is $90 \%$ or greater within $4.2-18.0 \mathrm{GHz}$ intervals. It is operational bandwidth is therefore 13.8 $\mathrm{GHz}$ with thickness of $4.2 \mathrm{~mm}$. The operational bandwidth to thickness ratio performance of the proposed RAMM is 13.029 . The proposed RAMM

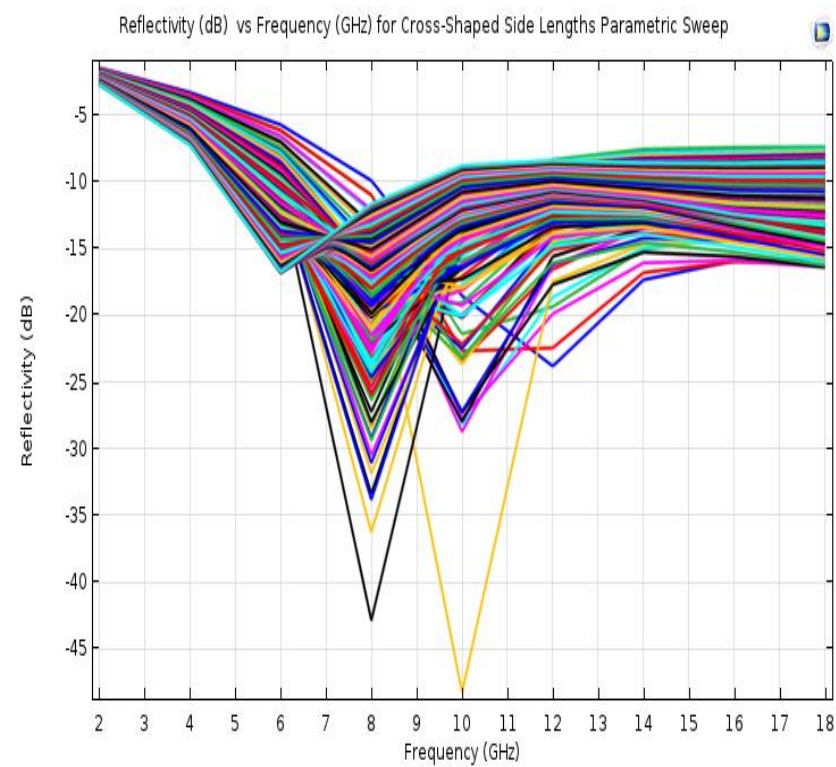

Figure 4: Cross-Shaped layer side lengths parametric sweep results thickness, bandwidth and operational bandwidth to thickness ratio indicated an outstanding performance towards overcoming the challenging task of having broadband and thin thickness absorber, compared to the currently published structures of its kind [27-30], [32], [34-36]. The below $-10 \mathrm{~dB}$ reflectivity bandwidth of $13.8 \mathrm{GHz}$ for the designed RAMM is wider than the $10 \mathrm{GHz}$ bandwidth obtained in [29], $13.2 \mathrm{GHz}$ in [30] and $3.5 \mathrm{GHz}$ in [32]. Others are $10 \mathrm{GHz}$ [34], $3.13 \mathrm{GHz}$ [35] and $3.18 \mathrm{GHz}$ [36]. Likewise, the referenced absorbers' operational bandwidth to thickness ratio of 7.204 [29], 9.745 [30] and 6.43 [32] are smaller than that of the modeled RAMM value of 13.029. The designed RAMM thus outclassed these broadband absorbers because the larger the ratio, the better the performance of the absorber [32].

For the calculated result, reflectivity below $-10 \mathrm{~dB}$ takes place from 3.1 to $18.0 \mathrm{GHz}$. Comparatively there is favorable agreement between the simulated and the calculated results. The results tend to be consistent in both shape and peaks, though there is deviation in respect of the working bandwidth $(13.8 \mathrm{GHz}$ for the simulated and $14.9 \mathrm{GHz}$ for the calculated). This could be attributed to the fact that, the middle and surface layers are not in full accord with honeycomb-structure employed in the theoretical assessment of the RAMM as their volume ratio is slightly greater than $50 \%$ [29]. Figure 9 shows the power loss density of the threelayered absorber at the frequency of $4 \mathrm{GHz}$ and Figure 10 revealed that of $12 \mathrm{GHz}$ in simulation.

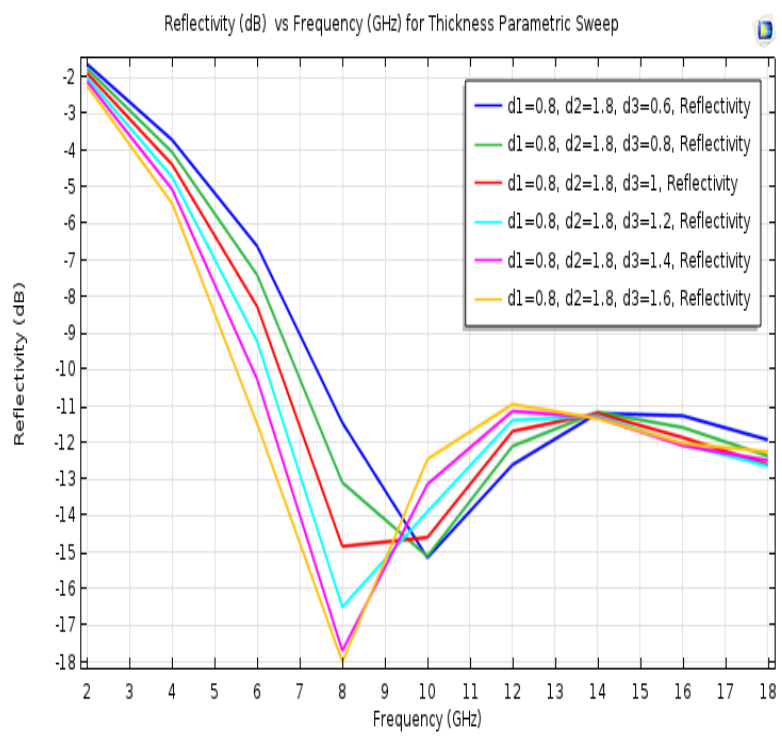

Figure 5: Layers thickness parametric sweep for RAMM 
It can be seen clearly that the main loss at $4 \mathrm{GHz}$ frequency of the incident wave occurred around the central area of the bottom layer, which simply corresponded to the surface layer as it is in alignment with the position of the cross-shaped surface layer. This contradicts the typical evenly horizontal loss in conventional slab [29] making the structure behaving in an unconventional manner hence becoming a metamaterial. It is therefore a convincing fact that the surface and middle layers provided appropriate impedance matching conditions where the full-wave energy of $2-8 \mathrm{GHz}$ frequency regime was consumed mainly in the bottom layer.

Meanwhile, the main loss at $12 \mathrm{GHz}$ happened at the central position of the cross-shaped surface layer structure as revealed in Figure 10. According to [37]; absorption characteristics of magnetic absorbing materials such as the carbonyl iron flakes used in this work, is weak in the $2-8 \mathrm{GHz}$ frequency range even though they can absorb more than $85 \%$ of the incident electromagnetic wave over $8-18 \mathrm{GHz}$ range.

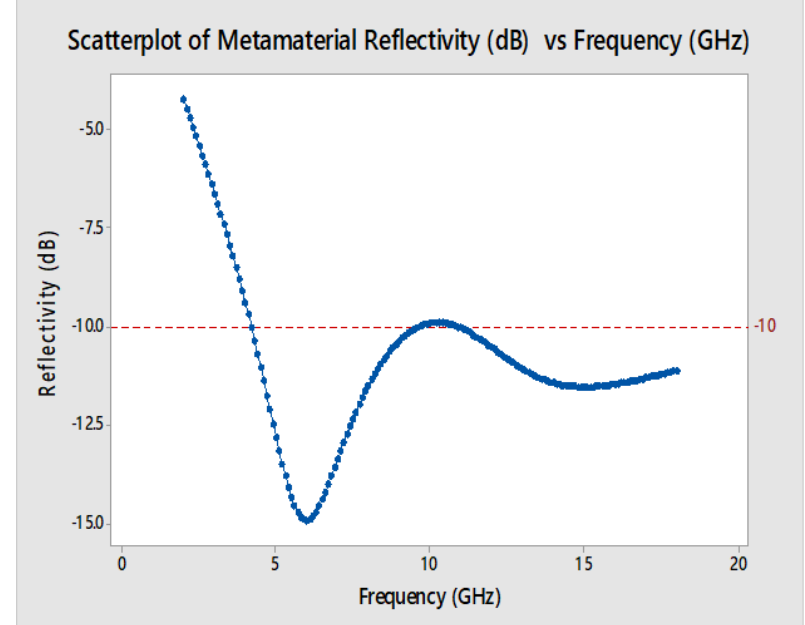

Figure 6: Reflectivity result obtained from the RAMM simulation on COMSOL Multiphysics

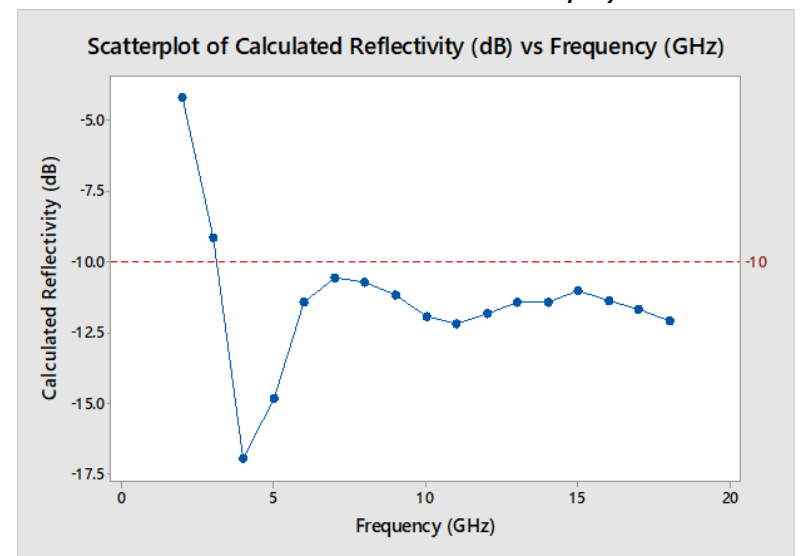

Figure 7: Calculated reflectivity result of the designed RAMM

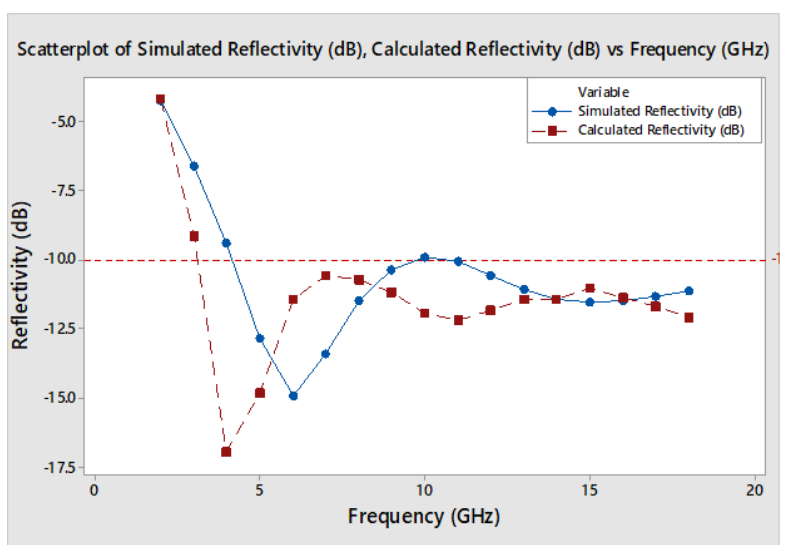

Figure 8: Simulation and Calculation results of the designed RAMM

freq $(21)=4 E 9$ Volume: Electromagnetic power loss density $\left(\mathrm{W} / \mathrm{m}^{3}\right)$

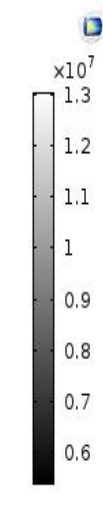

Figure 9: Electromagnetic Power Loss Density of the RAMM at $4 \mathrm{GHz}$

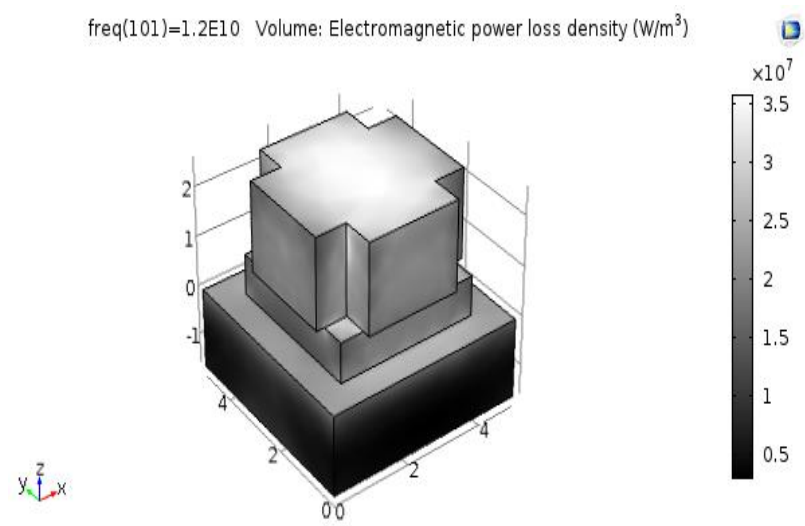

Figure 10: Electromagnetic Power Loss Density of the RAMM at $12 \mathrm{GHz}$

This is however improved by the structural design of the modeled RAMM which guides the $2-8 \mathrm{GHz}$ frequencies to the bottom layer for effective absorptions while the $8-18 \mathrm{GHz}$ frequencies are efficiently absorbed at the surface layer. Therefore, it is reasonable to infer that the absorption band of metamaterial at low frequencies and that of magnetic microwave absorbing materials at high frequencies are both inherited by the designed metamaterial absorber as clearly indicated by the electromagnetic power loss density results. 
Figure 11 provided the plots of simulated reflectivity of metamaterial and conventional absorbers made from the same material and thickness for performance comparison. From the figure, the designed metamaterial absorber clearly surpassed the conventional one as none of the entire $2-18 \mathrm{GHz}$ frequency range reaches $-10 \mathrm{~dB}$ reflectivity bench marks for good absorption characteristics.

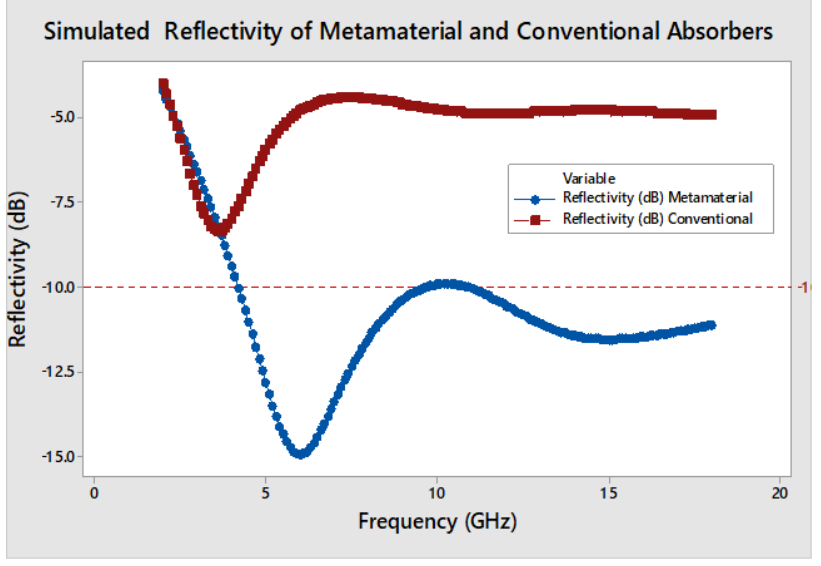

\section{CONCLUSION}

A new broadband radar absorbing metamaterial was developed in COMSOL Multiphysics environment. The designed RAMM demonstrates good absorption features with reflectivity below $\quad-10 \mathrm{~dB}(>90 \%$ absorption) from $4.2 \mathrm{GHz}$ to $18 \mathrm{GHz}$ in the radar frequency band having a total thickness of $4.2 \mathrm{~mm}$ only. Moreover, the proposed RAMM has relatively wider operational bandwidth compared to the currently published structures discussed, with a competitive value of 13.029 for the operational bandwidth to thickness ratio. The absorption bandwidth below $-10 \mathrm{~dB}$ is $14.9 \mathrm{GHz}$ for the calculated and $13.8 \mathrm{GHz}$ for the simulated and the closely resemble shapes and peaks of the simulated and calculated results further confirm the validity of the designed structure. The developed RAMM has potentials to be used for multiple electromagnetic applications like stealth technology and wireless communication.

\section{REFERENCES}

[1] V. G. Veselago, "The electrodynamics of substances with simultaneously negative values of $\varepsilon$ and $\mu$," Sov. Phys. Uspekhi, vol. 10, no. 4, pp. 509-514, 1968.

[2] J. B. Pendry, "Negative refraction makes a perfect lens," Phys. Rev. Lett., vol. 85, no. 18, pp. 3966-3969, 2000.

[3] D. R. Smith, "How to build a superlens," Science
(80-. )., vol. 308, no. 5721, pp. 502-503, 2005.

[4] D. Schurig et al., "Metamaterial electromagnetic cloak at microwave frequencies," Science (80-. )., vol. 314, no. 5801, pp. 977-980, 2006.

[5] X. Valentine, J.; Zhang, S.; Zentgraf, T.; UlinAvila, E.; Genov, D.A.; Bartal, G.; Zhang, "Threedimensional optical metamaterial with a negative refractive index," Nature, vol. 455, pp. 376-379, 2008.

[6] W. J. Padilla, D. N. Basov, and D. R. Smith, "Negative refractive index metamaterials," vol. 9, no. 7, pp. 28-35, 2006.

[7] V. M. Shalaev, "Optical Negative-Index Metamaterials," Nat. Photonics, vol. 1, pp. 41-48, 2007.

[8] C. M. Watts, X. Liu, and W. J. Padilla, "Metamaterial electromagnetic wave absorbers," Adv. Mater., vol. 24, no. 23, 2012.

[9] X. Liu, T. Tyler, T. Starr, A. F. Starr, N. M. Jokerst, and W. J. Padilla, "Taming the Blackbody with Infrared Metamaterials as Selective Thermal Emitters," Phys. Rev. Lett., vol. 107, no. 4, pp. 47, 2011.

[10] W. C. Chen, C. M. Bingham, K. M. Mak, N. W. Caira, and W. J. Padilla, "Extremely subwavelength planar magnetic metamaterials," Phys. Rev. B - Condens. Matter Mater. Phys., vol. 85, no. 20, pp. 1-5, 2012.

[11] N. I. Landy, S. Sajuyigbe, J. J. Mock, D. R. Smith, and W. J. Padilla, "Perfect metamaterial absorber," Phys. Rev. Lett., vol. 100, no. 20, pp. $1-4,2008$

[12] M. Cao and Q. Zhang, "Design of broadband multilayer metamaterial absorber," IEEE, vol. 18, pp. 8-10, 2018.

[13] Y. Pang, H. Cheng, Y. Zhou, Z. Li, and J. Wang, "Ultrathin and broadband high impedance surface absorbers based on metamaterial substrates," Opt. Express, vol. 20, no. 11, pp. 12515-12520, 2012.

[14] Y. Liu, X. Liu, and X. Wang, "Double-layer microwave absorber based on CoFe2O4 ferrite and carbonyl iron composites," J. Alloys Compd., vol. 584, pp. 249-253, 2014.

[15] Y. Danlée, I. Huynen, and C. Bailly, "Thin smart multilayer microwave absorber based on hybrid structure of polymer and carbon nanotubes," Appl. Phys. Lett., vol. 100, no. May, pp. 67-70, 2012.

[16] K. N. Rozanov, "Ultimate thickness to bandwidth ratio of radar absorbers," IEEE Trans. Antennas Propag., vol. 48, no. 8, pp. 1230-1234, 2000.

[17] X. Fan, J. Guan, W. Wang, and G. Tong, "Morphology evolution, magnetic and microwave 
absorption properties of nano/submicrometre iron particles obtained at different reduced temperatures," J. Phys. D. Appl. Phys., vol. 42, no. 7, p. 075006, 2009.

[18] X. Fan, J. Guan, Z. Li, F. Mou, G. Tong, and W. Wang, "One-pot low temperature solution synthesis, magnetic and microwave electromagnetic properties of single-crystal iron submicron cubes," J. Mater. Chem., vol. 20, no. 9, pp. 1676-1682, 2010.

[19] J. He, W. Wang, and J. Guan, "Internal strain dependence of complex permeability of ball milled carbonyl iron powders in $2-18 \mathrm{GHz}$," $J$. Appl. Phys., vol. 111, no. 9, pp. 1-6, 2012.

[20] L. Z. Wu et al., "High frequency complex permeability of iron particles in a nonmagnetic matrix," J. Appl. Phys., vol. 99, no. 083905, pp. 1-7, 2006.

[21] Y. B. Feng, T. Ã. Qiu, and C. Y. Shen, "Absorbing properties and structural design of microwave absorbers based on carbonyl iron and barium ferrite," J. Magn. Magn. Mater., vol. 318, no. 12, pp. 8-13, 2007.

[22] Z. Han et al., "Broadband electromagnetic-wave absorption by $\mathrm{FeCo} / \mathrm{C}$ nanocapsules," Appl. Phys. Lett., vol. 95, no. 2, pp. 9-12, 2009.

[23] Y. C. Qing, W. C. Zhou, F. Luo, and D. M. Zhou, "Microwave absorbing and mechanical properties of carbonyl iron powder/epoxy-silicon resin coatings," J. Magn. Magn. Mater., vol. 321, no. 1, pp. 25-28, 2009.

[24] O. Acher and S. Dubourg, "Generalization of Snoek's law to ferromagnetic films and composites," Phys. Rev. B - Condens. Matter Mater. Phys., vol. 77, no. 10, pp. 1-11, 2008.

[25] O. Acher and A. L. Adenot, "Bounds on the dynamic properties of magnetic materials," Phys. Rev. B - Condens. Matter Mater. Phys., vol. 62, no. 17, pp. 11324-11327, 2000.

[26] A. N. Lagarkov and K. N. Rozanov, "Highfrequency behavior of magnetic composites," $\mathrm{J}$. Magn. Magn. Mater., vol. 321, no. 14, pp. 20822092, 2009.

[27] W. S. Chin and D. G. Lee, "Development of the composite RAS (radar absorbing structure) for the X-band frequency range," Compos. Struct., vol. 77, no. 4, pp. 457-465, 2007.

[28] Y. Xu, W. Yuan, S. Bie, H. Xu, Q. Chen, and J. Jiang, "Broadband microwave absorption property of a thin metamaterial containing patterned magnetic sheet," J. Electromagn. Waves Appl., vol. 29, no. 18, pp. 2420-2427, 2015.

[29] D. Zhou, X. Huang, and Z. Du, "Analysis and Design of Multi-layered Broadband Radar Absorbing Metamaterial Using 3D Printing Technology- based Method," IEEE Antennas Wirel. Propag. Lett., vol. 25, no. 1, pp. 1-4, 2016.

[30] Y.-J. Xiong et al., "Structural broadband absorbing metamaterial based on threedimensional printing technology," Acta Phys. Sin., vol. 67 , no. 8 , pp. $084202-1$ to $084202-8$, 2018.

[31] D. Zhou, X. Huang, Z. Du, and Q. Wang, "Calculation and Analysis of the Effective Electromagnetic Parameters of Periodic Structural Radar Absorbing Material Using Simulation and Inversion Methods," Prog. Electromagn. Res. M, vol. 52, no. November, pp. 57-66, 2016.

[32] T. Beeharry, R. Yahiaoui, K. Selemani, and H. H. Ouslimani, "A co-polarization broadband radar absorber for RCS reduction," Materials (Basel)., vol. 11, no. 9, pp. 1-11, 2018.

[33] B. Mulla and C. Sabah, "Perfect metamaterial absorber design for solar cell applications," Waves in Random and Complex Media, vol. 25, no. 3, pp. 382-392, 2015.

[34] Y. Fan et al., "Ultra-thin and -broadband Microwave Magnetic Absorber Enhanced by Phase Gradient Metasurface Incorporation," $\mathrm{J}$. Phys. D. Appl. Phys., vol. 51, no. 21, pp. 1-7, 2018.

[35] Q. Chen, S. Bie, W. Yuan, Y. Xu, H. Xu, and J. Jiang, "Low frequency absorption properties of a thin metamaterial absorber with cross-array on the surface of a magnetic substrate," J. Phys. D. Appl. Phys., vol. 49, no. 42, p. 425102, 2016.

[36] W. Li et al., "Low frequency and broadband metamaterial absorber with cross arrays and a flaked iron powder magnetic composite," AIP Adv., vol. 8, no. 1, pp. 015318-1 to 015318-9, 2018.

[37] W. Li, T. Wu, W. Wang, J. Guan, and P. Zhai, "Integrating non-planar metamaterials with magnetic absorbing materials to yield ultrabroadband microwave hybrid absorbers," Appl. Phys. Lett., vol. 104, no. 2, pp. 16-21, 2014. 